Rapid Communications

\title{
Quantitative Live Single-cell Mass Spectrometry with Spatial Evaluation by Three-Dimensional Holographic and Tomographic Laser Microscopy
}

\author{
Ahmed Ali, *,** Yasmine Abouleila,*,** Sara Amer, *,** Rie Furushima,* Samy Emara,** \\ Sebastien EQuIs, $* * *$ Yann CoTTE, ${ }^{* * *}$ and Tsutomu MASUJIMA ${ }^{* \dagger}$ \\ *Quantitative Biology Center (QBiC), RIKEN, 6-2-3 Furuedai, Suita, Osaka 565-0874, Japan \\ **Faculty of Pharmacy, Misr International University, Km28 Ismailia Road, Cairo, Egypt \\ ***Nanolive SA, Chemin de la Dent d'Oche 1a, Lausanne 1024, Switzerland
}

\begin{abstract}
The locations and volumes of the contents of a single HepG2 cell were visualized under three-dimensional (3D) holographic and tomographic (HT) laser microscopy, colored by refractive index, not staining. After trapping the specific area of a target cell in a nanospray tip, quantification was performed by live single-cell mass spectrometry. Comparison of the HepG2 cells' before and after 3D-HT images allowed the inference of the precise volume and original location of the trapped cell contents. The total amount of a trapped molecule was estimated. The images also revealed morphological changes in the cell structure caused by the manipulation.
\end{abstract}

Keywords Quantitative live single-cell mass spectrometry, three-dimensional imaging, holographic and tomographic microscopy, quantitative single-cell analysis, HepG2 cell

(Received December 18, 2015; Accepted January 22, 2016; Published February 10, 2016)

The cell has a three-dimensional (3D) structure and its spatial arrangement is often very important to molecular mechanisms essential for life. In order to visualize 3D morphologies of cells, confocal laser imaging was developed. ${ }^{1}$ The method is, however, only applicable to fluorescence-probed molecules, ${ }^{2}$ which limits the observable number of molecules, and such artificial probing sometime perturbs normal molecular mechanisms. Cotte et al. applied holographic and tomographic irradiation to microscopy and finally innovated a threedimensional computed holographic and tomographic (HT) laser microscope. ${ }^{3}$ The laser beam that penetrates the cell at an angle experiences a delay in the phase of its beam, which is magnified and overlayed with reference beam to make a holographic image. The holograms at various angles then deconvoluted by tomographic algorithms to create a precise 3D cell image. The 3D-HT microscope can visualize 3D morphological aspects by contrasting refractive indexes observed by the laser monochromatic wavelength, making staining unnecessary.

We have developed live single-cell mass spectrometry, ${ }^{4-7}$ in which the contents of a single cell, usually picoliter level or less, are sucked by a nanospray tip (a sort of glass capillary needle) and fed directly into a mass spectrometer after the addition of an ionization solvent to the rear end of the tip. In this method, the exact amount sucked is unclear because it is such a tiny volume. Furthermore, 3D spatial location and identity of the contents are also ambiguous. Through the combination of these two techniques, 3D-HT microscopy and live single-cell mass spectrometry, greater 3D spatial resolution (X-Y-axis $0.18 \mu \mathrm{m}$ and $\mathrm{Z}$-axis $0.33 \mu \mathrm{m}$ ) and improved quantitative single-cell analysis is expected. The first trial of this combination and its

$\dagger$ To whom correspondence should be addressed.

E-mail: masujima@ riken.jp results are documented in this paper, and we think nextgeneration live single-cell mass spectrometry is quite promising. Human hepatocellular carcinoma cell line (HepG2) was cultured in Dulbecco's modified Eagle medium in addition to $10 \%$ fetal calf serum (FBS), $100 \mathrm{mg} / \mathrm{mL}$ penicillin, and $100 \mathrm{mg} / \mathrm{mL}$ streptomycin $\mathrm{G}$ in $35 \mathrm{~mm}$ glass bottom dishes at $37^{\circ} \mathrm{C}$ and $5 \% \mathrm{CO}_{2}$. HepG2 cells were positioned under the HT laser microscope, and the HT scan took $2 \mathrm{~s}$ to acquire one 3D image. Figure 1 shows the schematic principle of the HT laser microscope (3D Cell Explorer, Nanolive, SA, Switzerland). ${ }^{3}$

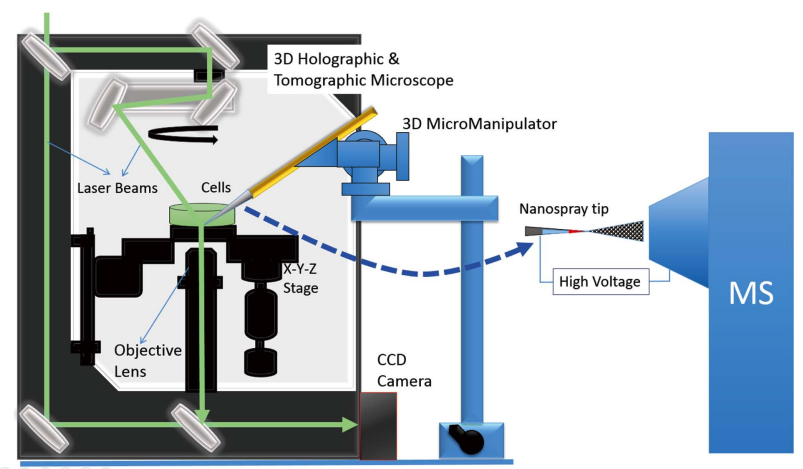

Fig. 1 Schematic of live single-cell mass spectrometry with quantitation by holographic and tomographic laser microscopy. The laser beam is split into a reference beam (going down to the CCD camera) and an observation beam that irradiates the cell at 45 degree angle. A micromanipulator was setup next to microscope to allow precise suction with a nanospray tip. The sucked cellular matter was then blasted through the mass spectrometer. 


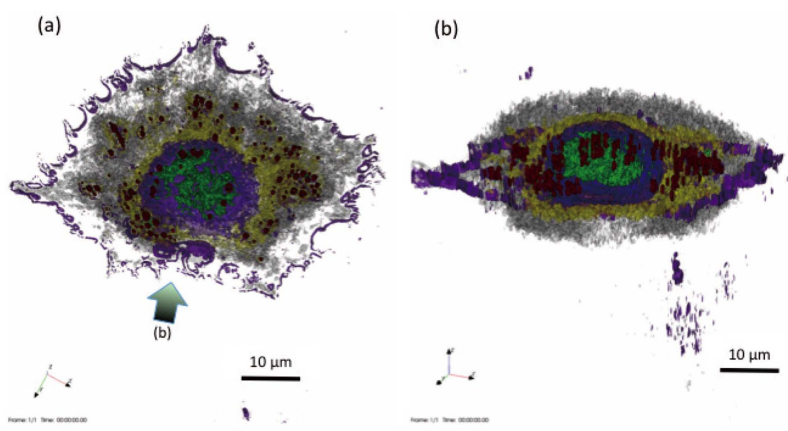

Fig. 2 Three-dimensional holographic and tomographic (3D-HT) microscopic images of a HepG2 cell; (a) top view and (b) side view from lower angle. Each color represents a different refractive index range. See text for more details.

The laser beam is split into a reference beam (going down to the camera) and an observation beam to irradiate the cell at an angle to get a hologram. The beam is rotated 360 degrees to allow the computed tomography for a very precise $3 \mathrm{D}$ image. To evaluate the cell volume, acquisition data were exported as image stack files, then analyzed using Fiji (Fiji is ImageJ). ${ }^{89}$ First, the 3D median filter was run to reduce background noise, then the cytoplasm volume was isolated using the color threshold tool. Moreover, the stack file was segmented using the segmentation editor to be reconstructed again digitally as a series of 96 stacks that have a defined geometric pattern in preparation for volume analysis. Finally, each stack thickness was calculated using the Object Counter plugin in Fiji. ${ }^{10}$

On the same metal base plate on which the HT laser microscope was standing, a micromanipulator was setup to suck out the contents of a single cell as shown in Fig. 1. For suction of single-cell contents, the microscope was set to usual bright field image mode. After the suction, the nanospray tip was removed from the manipulator, and an ionization solvent $(80 \%$ methanol, $10 \%$ dimethyl sulfoxide (DMSO), and $10 \mathrm{mmol}$ ammonium formate) was introduced from the back side of the tip to prepare for nanospray ionization for mass spectrometry. Molecular detection of the contents of a single HepG2 cell was performed using a high resolution mass spectrometer (LTQOrbitrap Velos Pro, Thermo Fisher Scientific) equipped with a nanospray ionization source. The spray voltage was applied around $1-1.5$ or -0.8 to $-1 \mathrm{kV}$, and the measuring $\mathrm{m} / \mathrm{z}$ range was set to 100 to 1000 , divided into increments of 50. Raw data were analyzed by MarkerView (Sciex) software in order to perform the peak alignment of the obtained spectra and the paired t-test analysis. Detected peaks were estimated by the exact $\mathrm{m} / \mathrm{z}$ value of $\pm 5 \mathrm{ppm}$ tolerance, and annotated by HMDB database (http://www.hmdb.ca/).

\section{Results and Discussion}

As shown in Fig. 2, the HepG2 cell has many granules (brown) around the central nucleus (purple) that contains chromatin (green). Almost all of these brown granules are mitochondria. The endoplasmic reticulum (yellow-green) is surrounding the nucleus. Judging from the side view, it is presumed that the cell adhered to the glass plate at its central region below the nucleus, not at the peripheral extended cytoplasm region which is, on the other hand, extending horizontally into the medium (Fig. 2(b)).

We took images before and after the suction of cytosol as seen in Fig. 3. The dashed line at the bottom of the image shows the

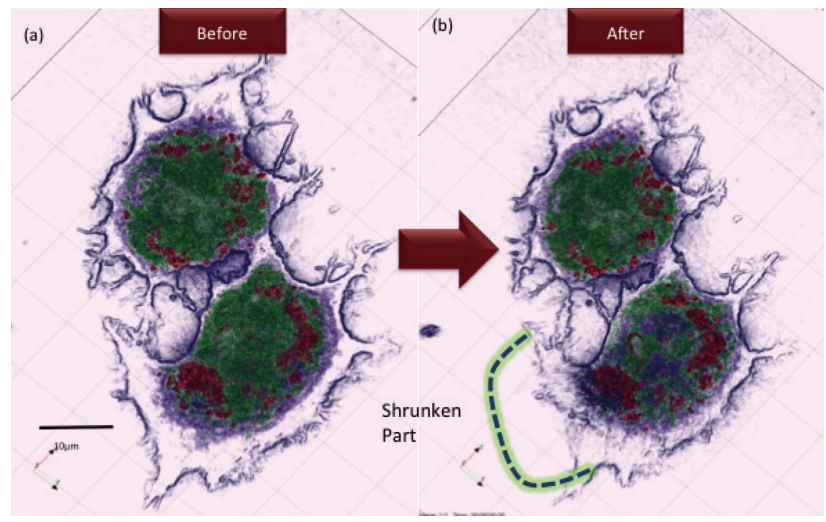

Fig. 3 HepG2 cell three-dimensional holographic and tomographic (3D-HT) image (a) before and (b) after insertion of the nanospray tip when a portion of the cell contents had been removed by suction. Cell morphology, colored by the individual refractive indexes of each component, is clearly visible. Comparison of the two 3D-HT images reveals the location and volume of the sucked contents.

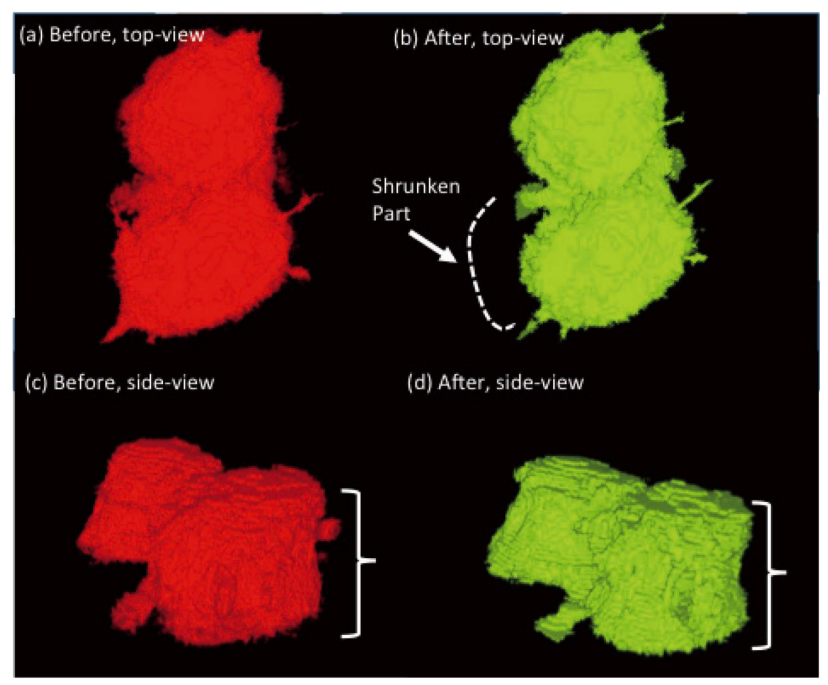

Fig. 4 Digital reconstruction of the acquisition data by the Fiji segmentation method in preparation for volume analysis. The data were segmented into 96 stacks and each stack thickness was calculated. Figures 4(a) and 4(b) show the difference in morphology and volume in the cell before and after suction. Figures 4(c) and 4(d) show the side view of the cell with emphasis on the change in the shape of the cell due to the suction.

cytoplasmic area affected by the suction, and the changes in the distributions of colors of the granules and nucleus can also be seen. Since the color is based on the refractive index, the morphology and positions of the granules and membranes seem to have been changed. The shrunken part due to suction and its volume can be estimated at $\pm 11 \mathrm{aL}$ accuracy.

Figure 4 shows the cell images during volume analysis. Using Fiji (ImageJ), the volume of a single granule was calculated to be $6.7 \mathrm{fL}$. By the geometrical stack segmentation method, the total difference in the volume of the cell before and after suction of cytosol region was calculated to be $1.16 \mathrm{pL}$. We were able to record the direct effects on the morphology and shape of the cell due to the suction of the nanospray tip as shown in Figs. 4(b) and 4(d). During the suction process, the cell itself is a little displaced and stretched vertically due to the vacuum effect of 


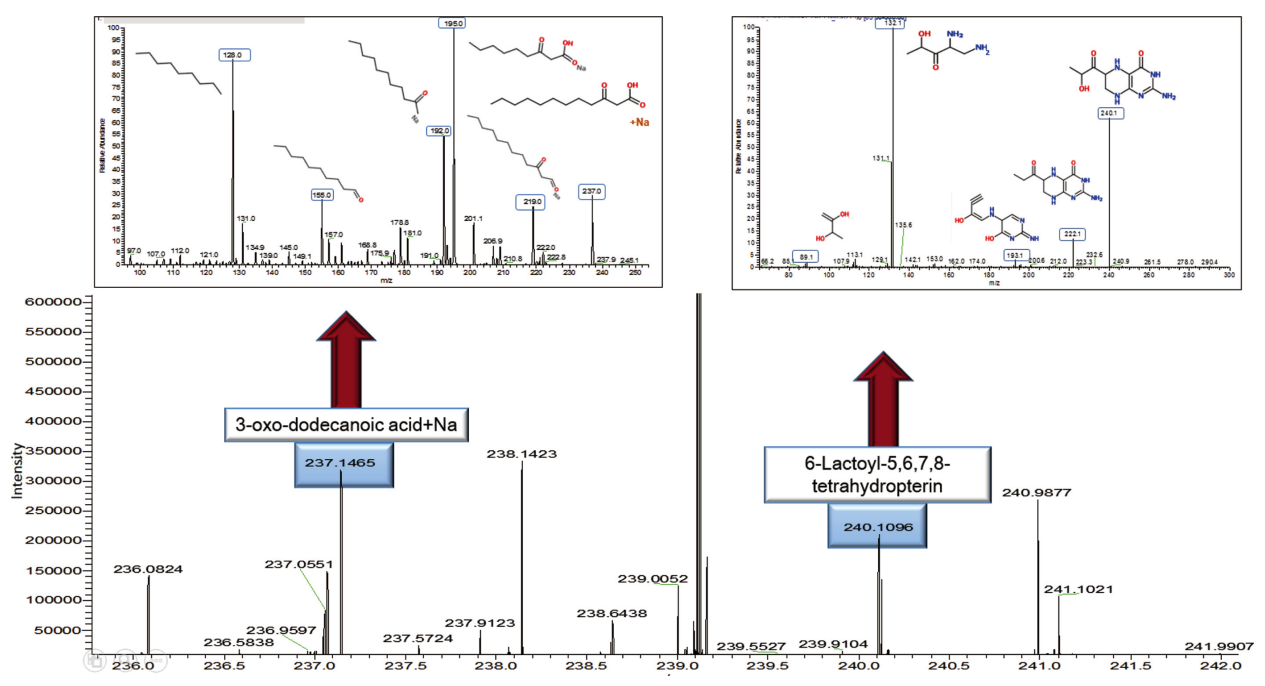

Fig. 5 Mass spectra of a HepG2 cell showing cytosol-specific peaks, along with their corresponding MS/MS spectra.

the nanospray tip as well as the movement of the tip upwards after sampling. The damage estimation of the cell is still not established, but accumulation of such data with membranespecific fluorescent probes will be useful for getting results.

Regarding mass spectrometric analysis, cytosol-specific molecules were detected in the spectra of sucked samples. We tried to quantify a cytosol-specific molecule, methionine sulfoxide $(\mathrm{m} / \mathrm{z}$ 166.0530). Using internal standard, stable isotope L-histidine- $15 \mathrm{~N} 3$, in the ionization solvent, we made a calibration curve and estimated the concentration of methionine sulfoxide in the trapped cytosol content to be $5.1 \mathrm{zmol} / \mathrm{pL}$ which is equal to $5.1 \mathrm{pmol} / \mathrm{mL}$. Since we trapped the content of $1.16 \mathrm{pL}$, thus total amount of trapped methionine sulfoxide was $5.9 \mathrm{zmol}$.

The other detected molecules include, but are not not limited to, glycolate $(\mathrm{m} / \mathrm{z}+\mathrm{K}$ 114.9793), choline $(\mathrm{m} / \mathrm{z}$ 104.1068), L-carnitine $(\mathrm{m} / \mathrm{z}$ 162.1129), L-sorbose $(\mathrm{m} / \mathrm{z}+\mathrm{Na}$ 203.053), arachidonic acid $(\mathrm{m} / \mathrm{z}+\mathrm{Na}$ 327.2301), D-glucosaminide $(\mathrm{m} / \mathrm{z}$ 502.2225), and diglyceride (16:0/20:3(5Z,8Z,11Z)/0:0) $(\mathrm{m} / \mathrm{z}$ 619.5297). In particular, compounds unique for liver cells were detected including: 6-lactoyl-5,6,7,8-tetrahydropterin $(\mathrm{m} / \mathrm{z} 240.1096$ and $+\mathrm{Na} 262.0918)$, B-N-acetyl glycosamine $(\mathrm{m} / \mathrm{z}, 222.0983), 5$-methyldeoxycytidine $(\mathrm{m} / \mathrm{z}, 242.1126)$, estrid3 -sulfate $(\mathrm{m} / \mathrm{z}+\mathrm{Na} 567.1519)$, 3-oxo-decanoic acid $+\mathrm{Na}(\mathrm{m} / \mathrm{z}$ 209.1151, 3-oxo-dodecanoic acid $+\mathrm{Na} 237.1465)$, 2-octenoic acid FA 8:0 $(\mathrm{m} / \mathrm{z}+\mathrm{Na} 165.089)$, and glucanic acid $(\mathrm{m} / \mathrm{z}+\mathrm{K}$ 249.001). As shown in Fig. 5, MS/MS was carried out for selected liver specific molecules and obtained fragment peaks were then identified using both HMDB (http://hmdb.ca), and MetFrag fragmentation databases (http://msbi.ipb-halle.de/ MetFrag/).

\section{Acknowledgements}

We thank Thermo Fisher Scientific Inc., especially Dr. A.
Makarov, for their support with scientific instruments, and Mrs. April Oga for assistance with writing this manuscript. This work was conducted under the RIKEN Pioneering Projects of "Single Cell Science" and "Biology of Symbiosis" and the fund for the Development of Advanced Measurement and Analysis Systems (SENTAN), by the Japan Agency for Medical Research and Development (AMED), and by the Japan Science and Technology Agency (JST) in 2013 - 2014.

\section{References}

1. G. J. Brakenhoff, P. Blom, and P. Barends, J. Microscopy, 1979, 117, 219.

2. Y. Hiraoka, T. Shimi, and T. Haraguchi, Cell Struct. Funct., 2002, 27, 367.

3. Y. Cotte, F. Toy, P. Jourdain, N. Pavillon, D. Boss, P. Magistretti, P. Marquet, and C. Depeursinge, Nature Photonics, 2013, 7, 113.

4. N. Tsuyama, H. Mizuno, E. Tokunaga, and T. Masujima, Anal. Sci., 2008, 24, 559.

5. H. Mizuno, N. Tsuyama, T. Harada, and T. Masujima, J. Mass Spectrum., 2008, 43, 1692.

6. T. Masujima, Anal. Sci., 2009, 25, 953.

7. T. Fujii, S. Matsuda, M. L. Tejedor, T. Esaki, I. Sakane, H. Mizuno, N. Tsuyama, and T. Masujima, Nat. Protoc., 2015, 10,1445 .

8. C. Schneider, W. Rasband, and K. Eliceiri, Nat. Methods, 2012, 9, 671.

9. J. Schindelin, I. Arganda-Carreras, and E. Frise et al., Nat. Methods, 2012, 9, 676.

10. S. Bolte and F. P. Cordelières, J. Microscopy, 2006, 224, 213. 